\title{
Colocalization of Tectal Inputs With Amygdala-Projecting Neurons in the Macaque Pulvinar
}

\author{
Catherine Elorette ${ }^{1,2}$, Patrick A. Forcelli ${ }^{1,2,3}$, Richard C. Saunders ${ }^{4}$ and Ludise Malkova ${ }^{1,2 *}$ \\ ${ }^{1}$ Interdisciplinary Program in Neuroscience, Georgetown University School of Medicine, Washington, DC, United States, \\ ${ }^{2}$ Department of Pharmacology and Physiology, Georgetown University School of Medicine, Washington, DC, United States, \\ ${ }^{3}$ Department of Neuroscience, Georgetown University School of Medicine, Washington, DC, United States, ${ }^{4}$ Laboratory of \\ Neuropsychology, National Institute of Mental Health (NIMH), Bethesda, ML, United States
}

OPEN ACCESS

Edited by: Tadashi Isa, Kyoto University, Japan

Reviewed by: Hisao Nishijo, University of Toyama, Japan

Paul J. May,

University of Mississippi Medical Center, United States

*Correspondence: Ludise Malkova malkoval@georgetown.edu

Received: 21 June 2018 Accepted: 03 October 2018 Published: 24 October 2018

Citation: Elorette C, Forcelli PA, Saunders RC and Malkova L (2018) Colocalization of Tectal Inputs With Amygdala-Projecting Neurons in the Macaque Pulvinar.

Front. Neural Circuits 12:91. doi: 10.3389/fncir.2018.00091
Neuropsychological and neuroimaging studies have suggested the presence of a fast, subcortical route for the processing of emotionally-salient visual information in the primate brain. This putative pathway consists of the superior colliculus (SC), pulvinar and amygdala. While the presence of such a pathway has been confirmed in sub-primate species, it has yet to be documented in the primate brain using conventional anatomical methods. We injected retrograde tracers into the amygdala and anterograde tracers into the colliculus, and examined regions of colocalization of these signals within the pulvinar of the macaque. Anterograde tracers injected into the SC labeled axonal projections within the pulvinar, primarily within the oral, lateral and medial subdivisions. These axonal projections from the colliculus colocalized with cell bodies within the pulvinar that were labeled by retrograde tracer injected into the lateral amygdala. This zone of overlap was most notable in the medial portions of the medial (PM), oral (PO) and inferior pulvinar (PI), and was often densely concentrated in the vicinity of the brachium of the SC. These data provide an anatomical basis for the previously suggested pathway mediating fast processing of emotionally salient information.

Keywords: subcortical, superior colliculus, blindsight, retrograde, anterograde, anatomy

\section{INTRODUCTION}

Threatening stimuli require fast detection and response from an organism. The canonical cortical visual processing stream refines information from coarse, low level representations in early cortical areas to detailed, high level information. The "trade off" for this refinement is that it requires many synaptic connections. Conserved patterns of low-level visual information, e.g., something moving quickly in peripheral vision, may be sufficient to serve as a threat detection pathway (Dean et al., 1989; Soares et al., 2017). On the basis of behavioral and anatomical studies some have suggested that low level or coarse visual information may travel to the forebrain in a rapid, subcortical manner (LeDoux, 1996; Silverstein and Ingvar, 2015).

Despite a lack of conscious recognition, patients with damage to primary visual cortex can retain appropriate responses to visual stimuli (Pöppel et al., 1973; Richards, 1973; Sanders et al., 1974; Weiskrantz et al., 1974). In a non-conscious manner, patients with so-called "blindsight" can detect, localize, and distinguish between stimuli that are presented within the field of vision damaged by a lesion (Cowey and Stoerig, 1991; Sahraie et al., 1998). Similarly, even after total destruction of V1, non-human primates can track moving stimuli and distinguish between different textures, colors, 
shape, or frequency of presentation of stimuli (Weiskrantz, 1963; Humphrey and Weiskrantz, 1967; Mohler and Wurtz, 1977; Miller et al., 1980). The preservation of visual processing in blindsight is subserved in part by projections from the retina to the lateral geniculate nucleus (LGN) of the thalamus (Warner et al., 2010). The LGN, which is the primary relay to visual cortex, projects not only to primary visual areas, but also to higher order cortical regions (e.g., V4, MT; Born and Bradley, 2005). These extrastriate projections are likely preserved after damage to V1 (Schmid et al., 2009). In addition, the superior colliculus (SC), which receives direct retinal input, may provide a relay for visual processing in the absence of V1. Accordingly, inactivation of either the LGN or the SC disrupts blindsight in macaques (Schmid et al., 2010; Kato et al., 2011; Takakuwa et al., 2017).

In addition to the preservation of these non-conscious visual abilities, processing of emotional information in patients with blindsight is likewise preserved. For example, patients can continue to discriminate between facial expressions (de Gelder et al., 1999) or fear-evoking images (Morris et al., 2001) after damage to V1. Furthermore, activation of the amygdala in response to emotionally salient visual stimuli has been observed using fMRI in cortically blind individuals (Vuilleumier et al., 2002). Consistent with the notion that rapid detection of threatening stimuli may rely on a fast (subcortical) relay, in intact individuals masked presentation of emotionally salient stimuli likewise activates the amygdala in the absence of conscious awareness (Whalen et al., 1998; Liddell et al., 2005). While the LGN likely mediates many features of blindsight as described above, other subcortical structures have been suggested to mediate the fast processing of emotionally salient information. Notably, both the SC and pulvinar display coincident activation with the amygdala during processing of emotional stimuli (Morris et al., 1999; Koller et al., 2018); together with the amygdala, these structures have been suggested to serve as a coarse, fast, subcortical pathway for the detection and response to emotionally salient visual information (Weiskrantz et al., 1974; LeDoux, 1996).

The SC in primates receives input from $\sim 10 \%$ of retinal ganglion cells, and detects stimuli both in the central and peripheral field of vision (Perry and Cowey, 1984; Hofbauer and Dräger, 1985); in this way the SC may be optimally suited for detection of approaching threats. While the SC does not project directly to the amygdala, there is a well-documented projection from the SC to the pulvinar (Gattass et al., 2018, for review). Both the superficial and deep layers of SC project to pulvinar, including to the inferior (Stepniewska et al., 2000) medial, lateral and oral regions (Benevento and Fallon, 1975; Trojanowski and Jacobson, 1975; Benevento and Standage, 1983). Likewise, a projection from the pulvinar to the amygdala has been described (Locke, 1960; Jones and Burton, 1976; Aggleton et al., 1980; Norita and Kawamura, 1980; Romanski et al., 1997; Stefanacci and Amaral, 2000) with the majority of this projection targeting the lateral nucleus. This finding is consistent with previous findings that the bulk of subcortical and cortical inputs in the amygdala enter through the lateral nucleus (Amaral et al., 1992), considered the main input nucleus of the amygdala.
Neuroimaging studies in both human and macaque subjects have shown probabilistic connectivity among SC, pulvinar and amygdala (Tamietto et al., 2012; Rafal et al., 2015) using diffusion tensor imaging (DTI) and tractography methods. However, it remains to be determined if the regions of the pulvinar that project to the amygdala are the same regions that receive input from the SC. In the present study, we aimed to address this gap. To achieve this goal, we injected anterograde tracers in the SC and retrograde tracers in the lateral nucleus of the amygdala and documented an overlap between retrograde and anterograde labeling within the pulvinar.

\section{MATERIALS AND METHODS}

\section{Subjects}

Two male macaque monkeys (1 Macaca mulatta (Y), 1 Macaca nemestrina (S)) were used for these experiments. Animal $\mathrm{Y}$ was 4 years old and weighed $8.2 \mathrm{~kg}$ and Animal S was 6 years old and weighed $17.4 \mathrm{~kg}$ at the time of surgery. MRI-guided injections of anatomical tracers were performed stereotaxically under sterile conditions. Injections sites, tracers used and volumes of injection for each case are shown in Table 1. The animals received bilateral injections of anterograde tracer (fluoroemerald, FE) in SC and retrograde tracer (choleratoxin $\mathrm{B}, \mathrm{CTB}$ ) in the lateral nucleus of the amygdala; this yielded four cases for anatomical analysis. This study was carried out in accordance with the recommendations of the Guide for the Care and Use of Laboratory Animals. The protocol was approved by the Georgetown University Animal Care and Use Committee.

\section{MRI, Surgery and Injections}

To obtain a pre-operative MRI scan, each animal was sedated with ketamine $(10 \mathrm{mg} / \mathrm{kg})$, intubated, and maintained at a stable plane of anesthesia using isoflurane (1\%-4\%). Animals were then transported to the imaging facility (Center for Molecular Imaging at Georgetown University Medical Center) where they were placed into a standard MRI-compatible stereotaxic frame. Each animal received a T1-weighted MRI scan using a custom surface coil in Siemens Trio 3T MRI scanner, as previously described (Wellman et al., 2005). An MPRAGE pulse sequence $(\mathrm{TR}=1,600 \mathrm{~ms}, \mathrm{TE}=4.38 \mathrm{~ms}$, $\mathrm{TI}=640 \mathrm{~ms}$, flip angle $=15$ degrees, averages $=3$, FOV $=256 \times 256 \mathrm{~mm}^{2}$ ) was used to acquire a $3 \mathrm{D}$ volume of the monkey brain with an effective resolution of $1.0 \mathrm{~mm}^{3}$. The resulting subject-specific

\begin{tabular}{lllll}
\hline \multicolumn{4}{l}{ TABLE 1 } & Tracer injections across cases. \\
\hline Case & Tracer & Supplier & Site & Volume $(\boldsymbol{\mu}$ l) \\
\hline Y1 & Choleratoxin B & List Labs & L Amygdala & 2 \\
& Fluoroemerald & Invitrogen & L SC & 2.3 \\
Y2 & Choleratoxin B & List Labs & R Amygdala & 2.5 \\
& Fluoroemerald & Invitrogen & R SC & 2.3 \\
S1 & Choleratoxin B & Invitrogen & L Amygdala & 2.6 \\
& AlexaFluor-594 & & & \\
& conjugate & & & \\
& Fluoroemerald & Invitrogen & L SC & 2 \\
S2 & Choleratoxin B & List Labs & R Amygdala & 1.8 \\
& Fluoroemerald & Invitrogen & R SC & 2
\end{tabular}


atlas was used to calculate injection coordinates relative to the earbar and the superior sagittal sinus, both of which were visible on the scans (Saunders et al., 1990).

After the MRI scan, each animal was transported to the surgical suite while remaining in the stereotaxic frame. During surgery, vital signs (heart rate, respiratory rate, body temperature, oxygen saturation, electrocardiogram and end-tidal $\mathrm{CO}_{2}$ ) were monitored. Body temperature was maintained using a heated table and blankets. Intravenous fluids (lactated Ringer's solution) were delivered during the surgery.

The skin and galea were opened in anatomical layers to expose the cranium. A midline craniotomy (approximately 4 by $6 \mathrm{~mm}$ ) was placed at the anteroposterior level of the intra-aural plane to expose the superior sagittal sinus, which served as the midline reference point. Craniotomies were placed above the amygdala and SC (three in total), the dura was incised, and a Hamilton syringe (30 gauge) was lowered to the MRI-derived coordinates. Injections were performed at a rate of $0.2 \mu \mathrm{L} / \mathrm{min}$, and the syringe was left in place for a minimum of $10 \mathrm{~min}$ following injection to prevent reflux of the tracer up the needle tract. At the conclusion of the injections, the dura, galea and skin were closed in anatomical layers. Post-operatively, animals received antibiotics, analgesics and dexamethasone $(0.5-1 \mathrm{mg} / \mathrm{kg})$ in consultation with the facility veterinarians.

\section{Tissue Preparation}

After a survival period of 14 days for Animal Y and 15 days for Animal S, the animals were deeply anesthetized with a sodium pentobarbital-based euthanasia solution and perfused transcardially with phosphate buffered saline followed by $4 \%$ paraformaldehyde. Brains were removed from the skull and post-fixed overnight in $4 \%$ paraformaldehyde. Following postfixation, brains were cryoprotected in a solution of $10 \%$ glycerol and $2 \%$ DMSO in phosphate buffer for $24 \mathrm{~h}$, then transferred to a 20\% glycerol, 2\% DMSO solution in phosphate buffer for $48 \mathrm{~h}$ (Rosene et al., 1986). Cryoprotected brains were blocked in the coronal plane and then flash frozen in $-80^{\circ} \mathrm{C}$ isopentane and stored at $-80^{\circ} \mathrm{C}$ until sectioning. The tissue was cut coronally in sections of $40 \mu \mathrm{m}$ thickness on a freezing stage sliding microtome (American Optical Model 860 Microtome Physitemp Instruments Inc., BFS-40MP Freezing stage).

\section{Histological Procedures}

Every 10th section from each brain was mounted onto gelatin subbed slides and air dried. The mounted sections were processed through a series of ethanol solutions, defatted, stained with thionin and cover slipped using DPX mountant (SigmaAldrich, St. Louis, MO, USA). These thionin stained sections were used to visualize the subdivisions of the thalamic nuclei, amygdala and SC.

To visualize CTB and FE labeling a series of sections was processed for each case through the pulvinar; adjacent sections were spaced by $\sim 400 \mu \mathrm{m}$. The antibodies, suppliers and dilutions used for immunohistochemical and immunofluorescent staining procedures are listed in Table 2.

\section{Immunofluorescence}

The sections were washed in phosphate-buffered saline (PBS) containing $0.3 \%$ TX-100 three times for $5 \mathrm{~min}$ and placed in blocking solution with agitation for $1 \mathrm{~h}$. Blocking solution consisted of $2 \%$ bovine serum albumin, 3.75\% normal goat serum, $0.3 \%$ TX-100, all in PBS. Primary antibodies (anti-CTB and anti-FE, described in Table 2), were added to the blocking solution and the tissue was incubated for an additional $48 \mathrm{~h}$ at $4^{\circ} \mathrm{C}$. The sections were washed five times for $5 \mathrm{~min}$, then placed in secondary antibody solution (donkey anti-goat Alexa 594 and donkey anti-rabbit Alexa 488, described in Table 2), which consisted of $2 \%$ BSA, $0.3 \%$ TX-100, and 3.75\% normal goat serum, all in PBS. The sections were incubated in secondary antibody for $90 \mathrm{~min}$ with agitation at room temperature. Following incubation in secondary antibody, sections were washed with PBS five times for 5 min each, mounted on gelatincoated slides, briefly air dried, and coverslipped with Vectashield hard set with DAPI (Vector Laboratories). This process was used for cases Y1, Y2 and S2. In case S1, two 1-in-10 series through the pulvinar were stained separately. In one series, axons projecting from the SC to the pulvinar were visualized via immunofluorescence as described above, using only the antibodies (anti-FE) corresponding to the tracer injected in SC. The second series was processed for immunohistochemistry (described below).

For all cases, sections through the amygdala and SC were processed as described above to visualize the injection sites. The procedure was the same as that described for immunofluorescence above, but only the antibodies that corresponded to the injection sites were used (anti-CTB in the amygdala and anti-FE in the SC).

\section{Immunohistochemistry}

For one case (S1), visualization of the CTB was enhanced using immunohistochemistry. Sections were washed in PBS containing $0.3 \%$ Triton X-100 (TX-100) three times for $5 \mathrm{~min}$, quenched in $0.6 \%$ peroxide and PBS solution for $10 \mathrm{~min}$, and placed in blocking solution with agitation for $1 \mathrm{~h}$. Blocking solution consisted of $2 \%$ bovine serum albumin, 3.75\% normal goat serum, 0.3\% TX-100, all in PBS. Primary antibody (anti-CTB,

TABLE 2 | Antibodies used for immunofluorescence and immunohistochemistry.

\begin{tabular}{|c|c|c|c|c|c|}
\hline Antibody & Supplier Catalog \# & Type & Host & Dilution & Immunogen \\
\hline Anti-CTB & List Biological \#703 & Polyclonal & Goat & $1: 3,200$ & B subunit (choleragenoid) \\
\hline Anti-Goat AlexaFluor-594 conjugated secondary & Jackson Immunoresearch \#705-586-147 & $\lg G(H+L)$ & Donkey & $1: 2,000$ & Goat lgG \\
\hline Anti-goat HRP-conjugated secondary & Jackson Immunoresearch \#705-005-147 & $\lg G(H+L)$ & Donkey & $1: 200$ & Goat lgG \\
\hline Anti-FE & ThermoFisher \#A-11095 & Polyclonal & Rabbit & $1: 4,000$ & Fluorescein \\
\hline Anti-rabbit AlexaFluor-488 conjugated secondary & Jackson Immunoresearch \#705-545-003 & $\lg G(H+L)$ & Donkey & $1: 2,000$ & Rabbit lgG \\
\hline
\end{tabular}


described in Table 2) was added to the blocking solution and the tissue was incubated for an additional $48 \mathrm{~h}$ at $4^{\circ} \mathrm{C}$. The sections were washed five times for $5 \mathrm{~min}$, then placed in secondary antibody solution (Biotin-SP conjugated donkey anti-goat, described in Table 2) and incubated with agitation for $90 \mathrm{~min}$ at room temperature. These sections were washed five times for $5 \mathrm{~min}$ in PBS, placed in ABC solution (Vector Labs) for $90 \mathrm{~min}$ with agitation, washed five times for $5 \mathrm{~min}$, and placed in diaminobenzidine tetrachloride (DAB, Sigma Aldrich, St. Louis, MO, USA) solution for $10 \mathrm{~min}$ with agitation. Then $0.025 \%$ peroxide was added and the sections were allowed to stain for approximately $3.5 \mathrm{~min}$ until there was visible contrast in the tissue. The sections were then washed with PBS five times, mounted on gelatin-coated slides, air-dried and dehydrated through ascending concentrations of ethanol before being cleared in xylenes and coverslipped with DPX.

\section{Microscopy and Data Analysis}

To outline the boundaries of individual subdivisions of the pulvinar, amygdala, and SC, we used thionin stained sections from a normal macaque brain (Figure 1). These sections were photographed using a Nikon NiE-E research microscope. Images were uniformly edited for brightness and noise reduction. The boundaries of amygdala nuclei and layers of the SC follow the divisions of Amaral et al. (1992) and May (2006), respectively. The boundaries of thalamic subnuclei were drawn following the conventions of the macaque thalamus atlas of Olszewski (1952). For further discussion of inferior pulvinar (PI) divisions, see Gutierrez et al. (1995).

To document the sites of tracer injections, immunofluorescent sections through the amygdala and the SC were photographed using a stereo microscope (Omano, China). Resulting micrographs were adjusted for brightness and contrast in Photoshop CC 2017 (Adobe) and are presented in Figure 2 together with drawings of matching sections from a standard rhesus macaque brain atlas generated in the Laboratory of Neuropsychology (LN), at the National Institute of Mental Health (NIMH).

Using sections processed for immunofluorescence (cases Y1, Y2, S2), labeled cell bodies and fibers (Figures 3, 4, 6) were
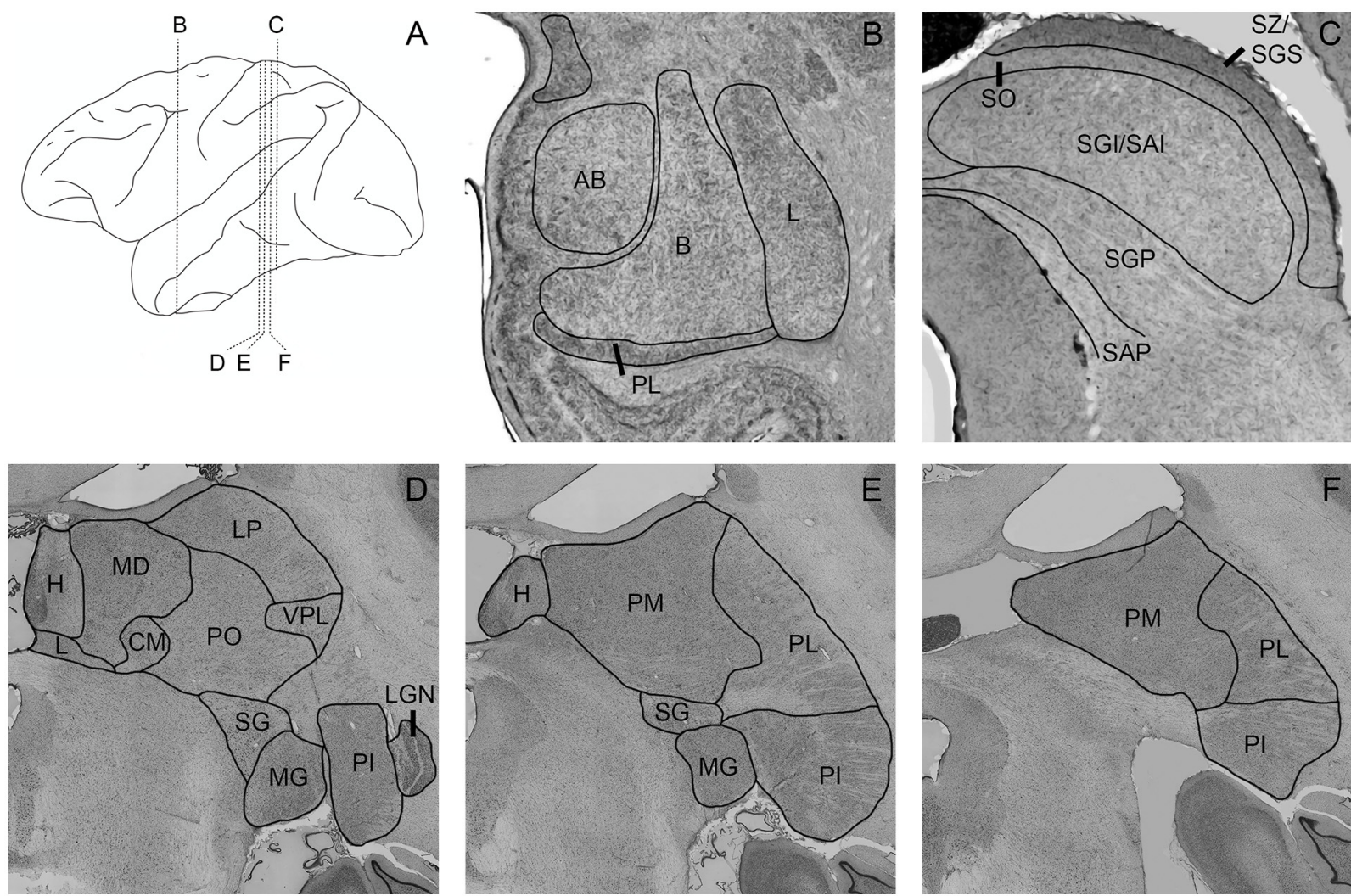

FIGURE 1 | Photomicrographs of nissl-stained sections through the amygdala, superior colliculus (SC), and pulvinar. Panel (A) shows the location of panels (B-F) within the brain. Amygdala nuclei (B) are outlined and labeled according to Amaral et al. (1992). AB, accessory basal; B, basal; L, lateral; M, medial; PL, paralaminar. SC nuclei (C) are outlined and labeled according to May (2006). SZ/SGS, stratum zonale/stratum griseum superficiale; SO, stratum opticum; SGI/SAI, stratum griseum intermedium/stratum album intermedium; SGP, stratum griseum profundum; SAP, stratum album profundum. Thalamic nuclei (D-F) are outlined and labeled according to the delineations of the Olszewski (1952). CM, centromedial nucleus; $H$, habenula; L, nucleus limitans; LGN, lateral geniculate nucleus; LP, lateral posterior nucleus; MD, mediodorsal nucleus; MG, medial geniculate nucleus; PI, inferior pulvinar; PL, lateral pulvinar; PM, medial pulvinar; PO, oral pulvinar; SG, suprageniculate nucleus; VPL, ventroposteriolateral nucleus. 

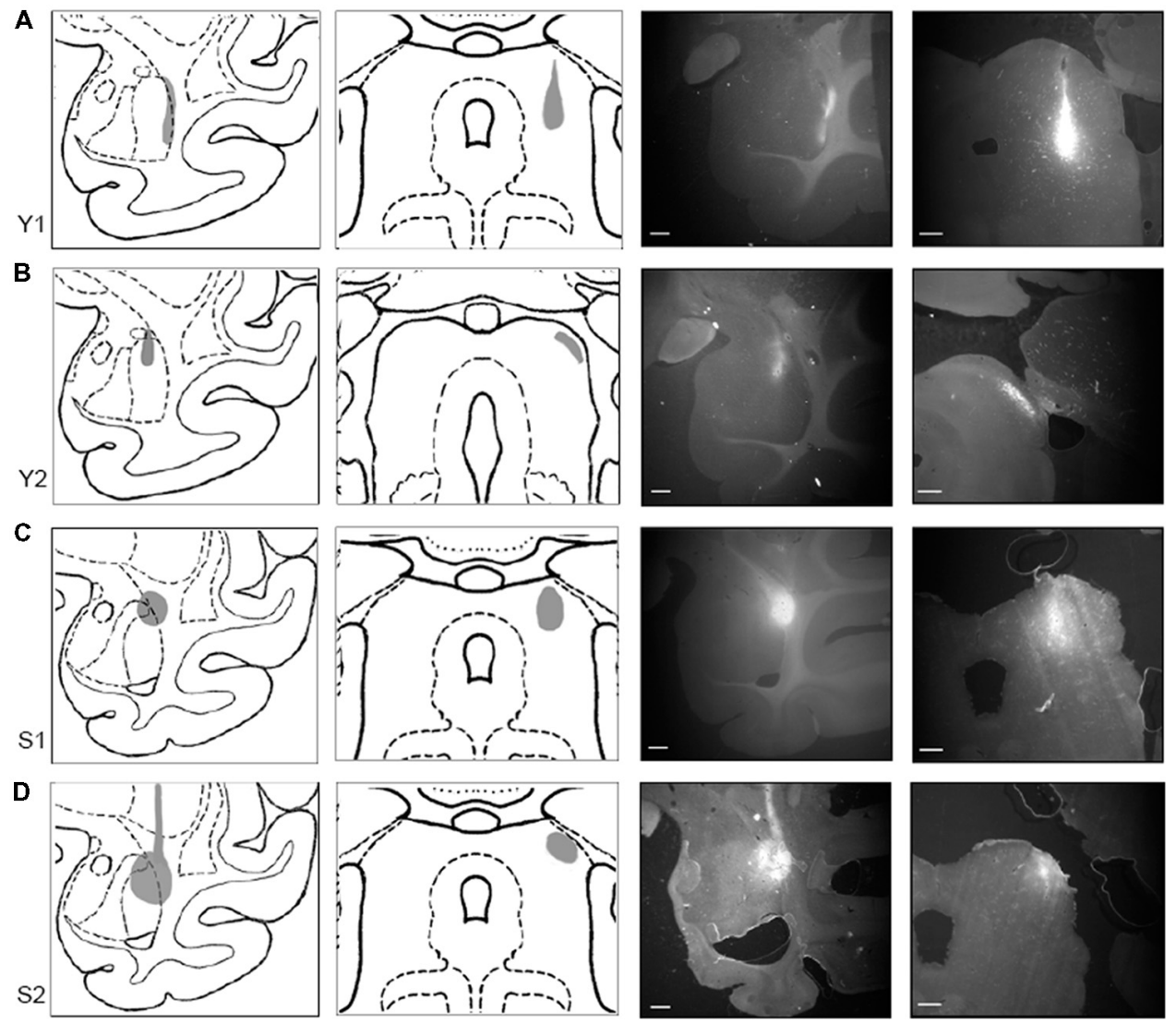

FIGURE 2 | Tracer injections localized in SC and amygdala. Injection sites for cases Y1 (A), Y2 (B), S1 (C), and S2 (D). Each injection site is shown (in gray) on drawings of coronal sections through a standardized rhesus macaque atlas at the level of the amygdala (1st column) and the SC (2nd column). Images on the right show photomicrographs of the fluorescent tracer injections in amygdala (choleratoxin B (CTB); 3rd column; scale bar = $5 \mathrm{~mm}$ ) and SC (fluoroemerald (FE); 4th column; scale bar $=1 \mathrm{~mm})$.

localized using a Zeiss Axiophot microscope fitted with an MDPlot digitizer and software (Accustage, Shoreview, MN, USA). The outline of each section was traced and the individual axons and cell bodies labeled with the different tracers were plotted. Cell bodies labeled with CTB within the pulvinar were differentiated from non-specific background signal on the basis of distinctive color, luminance and morphology. Labeled cells were brighter than background signal and had a characteristic nuclear exclusion of fluorescence. The plotted sections were exported to Illustrator CS (Adobe) and aligned with images of the adjacent thionin stained section. In case $S 1, D A B$ staining of cell bodies in the pulvinar and fluorescent staining of axons from SC were marked as described above, then overlaid onto each other. These merged images were overlaid onto their corresponding thionin sections (Figure 5). In all figures, the plotted sections were cropped to show only the relevant regions. Labeling outside of the thalamus is not shown.

Sections through the pulvinar containing both retrogradely labeled cell bodies from tracers in the amygdala and axons labeled by anterograde tracers placed in the SC were acquired using a confocal microscope (Leica SP8) with $20 \times$ and $63 \times$ lenses (Leica HC PL APO $20 \times / 0.75$ IMM CORR CS2, Leica
HC PL APO $63 \times / 1.40$ OIL CS2). Z-stacks were acquired for each region of interest, and maximal Z-projections were then pseudocolored and adjusted for brightness and contrast using ImageJ. Brightness and contrast were separately adjusted for each channel and each image, but all processes were applied to the entire image. Representative examples are presented in Figure 7 .

\section{RESULTS}

\section{Injection Sites}

In Case Y1 (Figure 2A), the CTB injection site in the amygdala extended along the lateral border of the lateral nucleus. Tracer extended into the white matter at the dorsal aspect of the injection. The dorsoventral extent of this injection spanned the majority of the lateral nucleus. The corresponding injection site in SC was localized to the lateral portion of stratum griseum intermedium (SGI), stratum album intermedium (SAI), stratum griseum profundum (SGP) and stratum album profundum (SAP). Together, these layers constitute the intermediate and deep layers of the SC. This injection site was the most ventral of the four cases. 

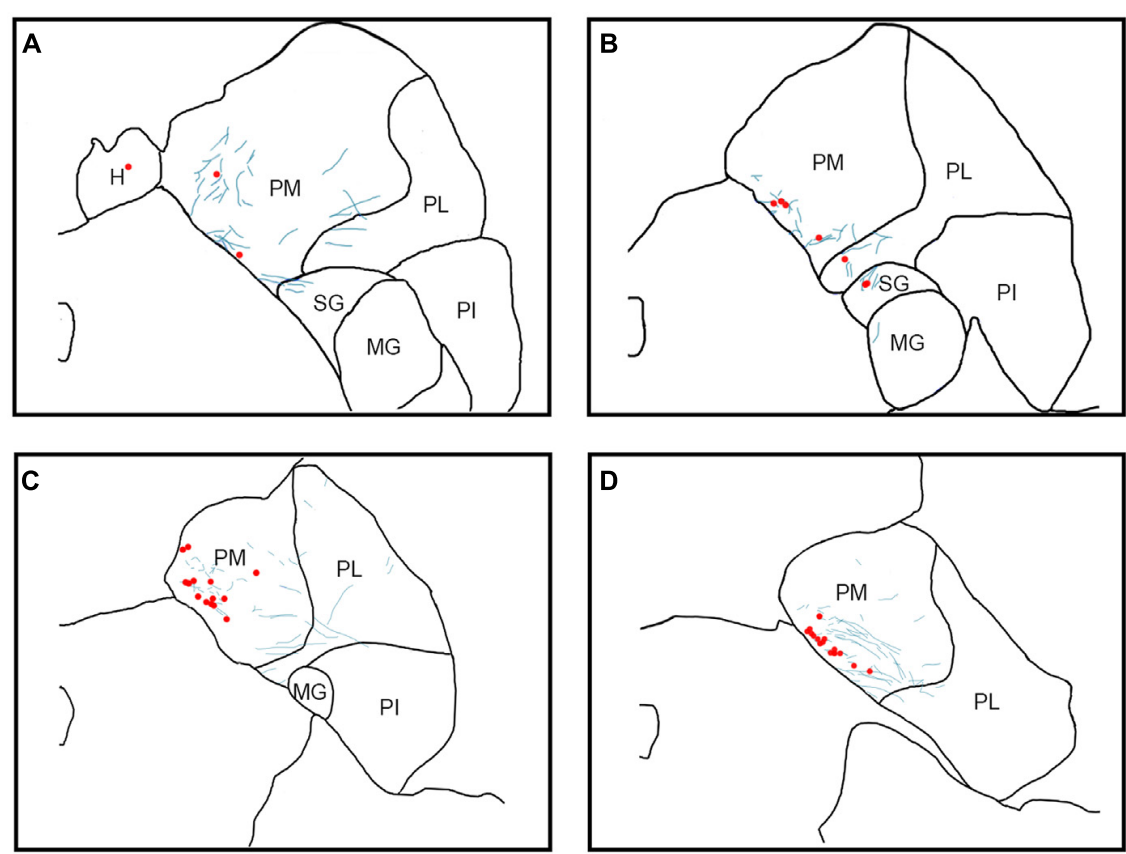

FIGURE 3 | Pattern of overlapping label in Case Y1. Each panel (A-D) presents a plot of labeled cell bodies or fibers from different rostro-caudal levels through the pulvinar. Anterograde label (putative axons) after injection of FE in the SC are shown in cyan. Retrograde label (cell bodies) after injection of CTB in the amygdala are shown in red. Abbreviations for thalamic nuclei are as in Figure 1.

In Case Y2 (Figure 2B), the CTB injection site in the amygdala was localized primarily to the dorsal portion of the lateral nucleus. The corresponding injection site in the SC was localized to the lateral stratum opticum (SO), with some tracer extended in to the stratum griseum superficiale (SGS) and SGI. The mediallateral spread of the tracer was limited with most the tracer located immediately lateral to the brachium.

In Case S1 (Figure 2C), the CTB injection site in the amygdala was localized to the dorsolateral portion of the lateral basal nucleus and the dorsal portion of the lateral nucleus. The injection site extended beyond the boundary of the amygdala into the laterally adjacent white matter. The FE SC injection site was centered on the intermediate layers of SC with tracer extending into the superficial (SO, SGS) and stratum zonale (SZ) and deep layers. The SC injection site in this case was the most medial of the four cases.

In Case S2 (Figure 2D), the CTB injection site in amygdala was centered in the dorsal aspect of the lateral nucleus. The injection covered the dorsal half of the lateral nucleus and extended medially into the dorsal part of the lateral basal nucleus, with some coverage of the lateral adjacent white matter. The SC injection site was localized to the lateral SC and extended throughout the superficial and intermediate layers. The injection site, while lateral, did not extend into the brachium.

\section{Localization of Anterograde and Retrograde Label Within the Pulvinar}

In Case Y1 (Figure 3), labeling of SC axons extended through the SC and spread through the medial pulvinar
(PM), lateral pulvinar (PL) and suprageniculate (SG). Several fiber bundles followed along the brachium of the SC, with the lateral extent along the borders of the thalamic nuclei. Retrogradely labeled cell bodies from injections in the amygdala were present along the brachium, in the PM, the PL, and within the SG nucleus, and were concentrated to the ventromedial aspects of these regions. Areas of both anterogradely labeled axons (from SC) and retrogradely labeled cell bodies were observed medially, in medial and (sparsely) in PL (Figures 3B-D, 7A).

In Case Y2 (Figure 4), labeled axons from anterograde injections in SC extended through the SC into the oral pulvinar (PO) and mediodorsal thalamus, and further extended to the lateral posterior (LP) and ventroposteriolateral (VPL) nuclei of the thalamus. These fibers were localized to the ventromedial PM and the lateral portion of the mediodorsal thalamus. Retrogradely labeled cell bodies were present in PO, PM, PI and nucleus limitans. These labeled cells were located in close proximity to the brachium, although a few cells were observed in the dorsal PO. Areas of overlapping anterograde and retrograde labeling were observed in oral, medial and PI, concentrated ventromedially in these regions (see Figure $\mathbf{7 B}$ for an example of labeling within PI).

In Case S1 (Figure 5), labeled axons extended through the SC and laterally from the brachium into medial, lateral and PI. The tracts largely appeared to travel mediolaterally and were densest in the ventral aspect of the PM and the lateral aspect of the PI. Retrogradely labeled cell bodies resulting from injection in the amygdala were present in medial and 

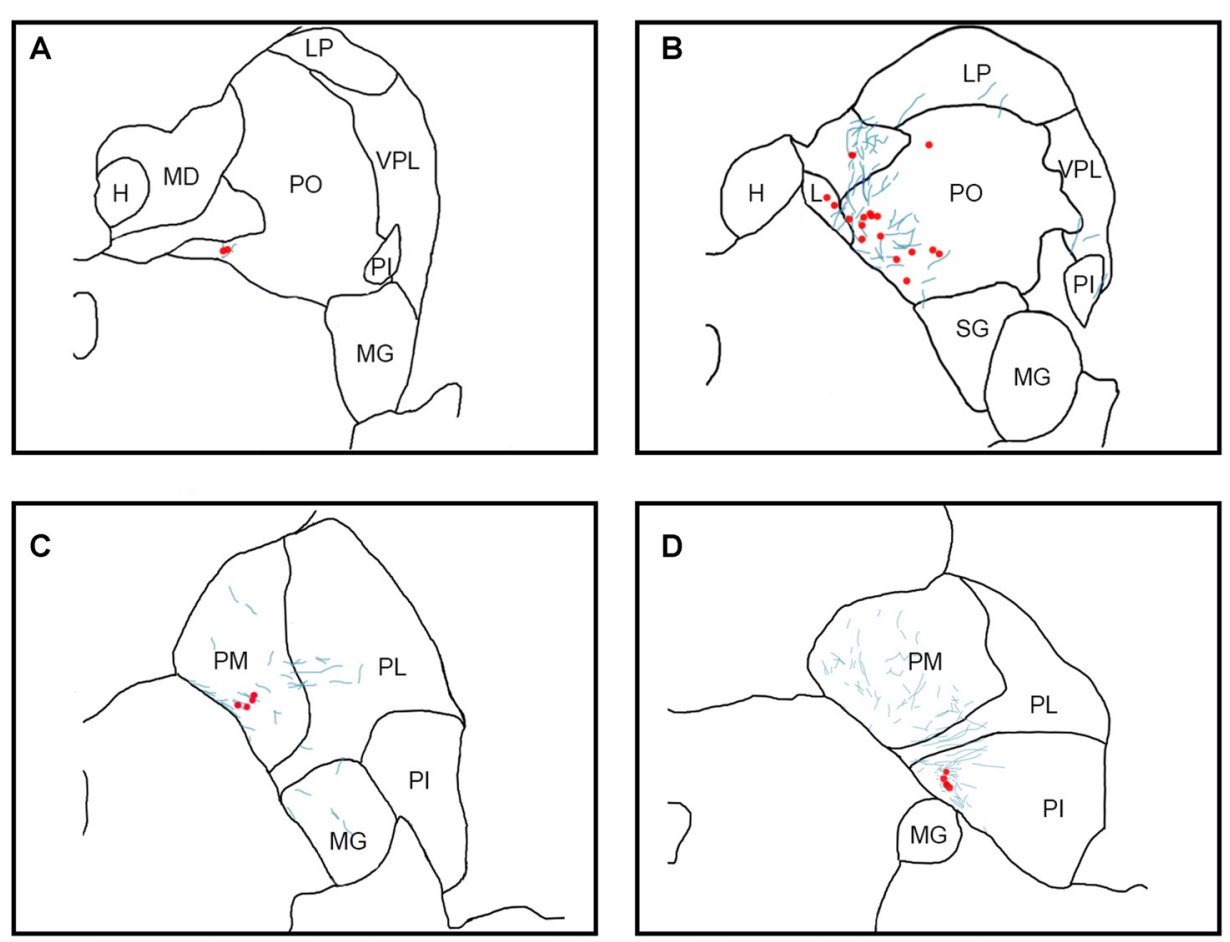

FIGURE 4 | Pattern of overlapping label in Case Y2. Each panel (A-D) presents a plot of labeled cell bodies or fibers from different rostro-caudal levels through the pulvinar. Anterograde label (putative axons) after injection of FE in the SC are shown in cyan. Retrograde label (cell bodies) after injection of CTB in the amygdala are shown in red. Abbreviations for thalamic nuclei are as in Figure 1.

$\mathrm{PL}$, and to a lesser extent within the SG/medial geniculate (MG) nucleus. Most cell bodies were ventromedially localized, but there were a large number present in PM that extended dorsoventrally to cover approximately half of the area of the nucleus. Areas of overlap between the labeled axons and cell bodies were not directly observed due to the type of staining utilized in this case (see "Materials and Methods" section), but both labeled axons from the SC and labeled neurons projecting to the amygdala were present in the ventromedial aspect of the PM. The more extensive retrograde signal in this case may be due to the better signal-to-noise of the DAB-based immunohistochemistry as compared to immunofluorescence
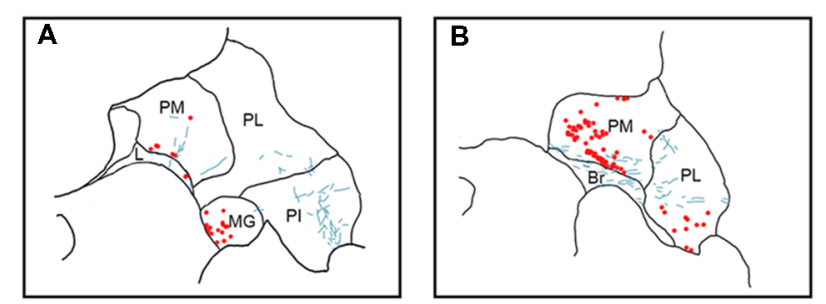

FIGURE 5 | Pattern of overlapping label in Case S1. Each panel $(\mathbf{A}, \mathbf{B})$ presents a plot of labeled cell bodies or fibers from different rostro-caudal levels through the pulvinar. Anterograde label (putative axons) after injection of FE in the SC are shown in cyan. Retrograde label (cell bodies) after injection of CTB in the amygdala are shown in red. Abbreviations for thalamic nuclei are as in Figure 1. Br, brachium of the superior colliculus. used in other cases. Since in this case, fluorescent CTB was used in one hemisphere and non-fluorescent CTB in the contralateral hemisphere, we were able to evaluate whether labeling was present only ipsilaterally or if it was also present contralaterally. In this case, labeling in the pulvinar appeared to be only ipsilateral.

In Case S2 (Figure 6), labeled axons extended from SC along the brachium, through the oral, medial and PL, as well as through central and parafascicular nuclei of the thalamus. Labeled axons were most densely concentrated in oral and PM, in the ventromedial aspect (see Figure 7D for high power magnification of an area in ventromedial PO). Retrogradely labeled cell bodies resulting from the amygdala injection were present in oral, medial and PL and the MG nucleus. Likewise, the pattern of labeling was ventromedial across the thalamus, with most cell bodies appearing in small clusters. Areas of overlap between these labeled axons and cell bodies were found within oral, medial and PL and MG. The area of most dense overlap was caudally distributed within the PM.

In summary, within the pulvinar, retrogradely labeled cell bodies were often observed in close proximity to anterogradely labeled axons. In all cases, the labeling of the cell bodies in the pulvinar after amygdala injections was sparse, suggesting it is a small projection. The areas where the retrograde label overlapped with anterograde axonal label from the SC injections were quite limited. While the oral and PM showed the largest degree of overlapping labeling, some overlap was 

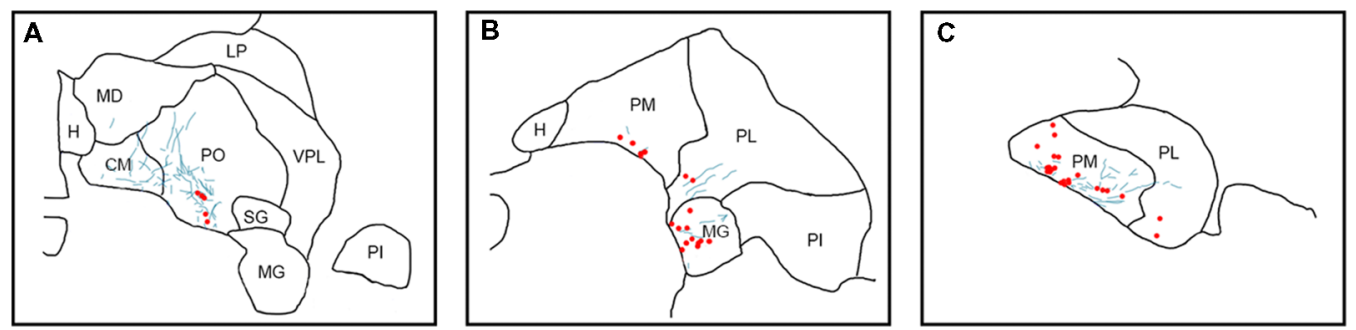

FIGURE 6 | Pattern of overlapping label in Case S2. Each panel (A-C) presents a plot of labeled cell bodies or fibers from different rostro-caudal levels through the pulvinar. Anterograde label (putative axons) after injection of FE in the SC are shown in cyan. Retrograde label (cell bodies) after injection of CTB in the amygdala are shown in red. Abbreviations for thalamic nuclei are as in Figure 1.

also observed in the lateral and PI, but to a lesser degree. Confocal imaging was used to extend these observations. Figure 7 shows images within the pulvinar from cases Y1,
Y2 and S2. Choleratoxin-positive cell bodies (pseudocolored red) and their processes within the pulvinar were found within a field of FE-labeled axons (pseudocolored cyan) resulting
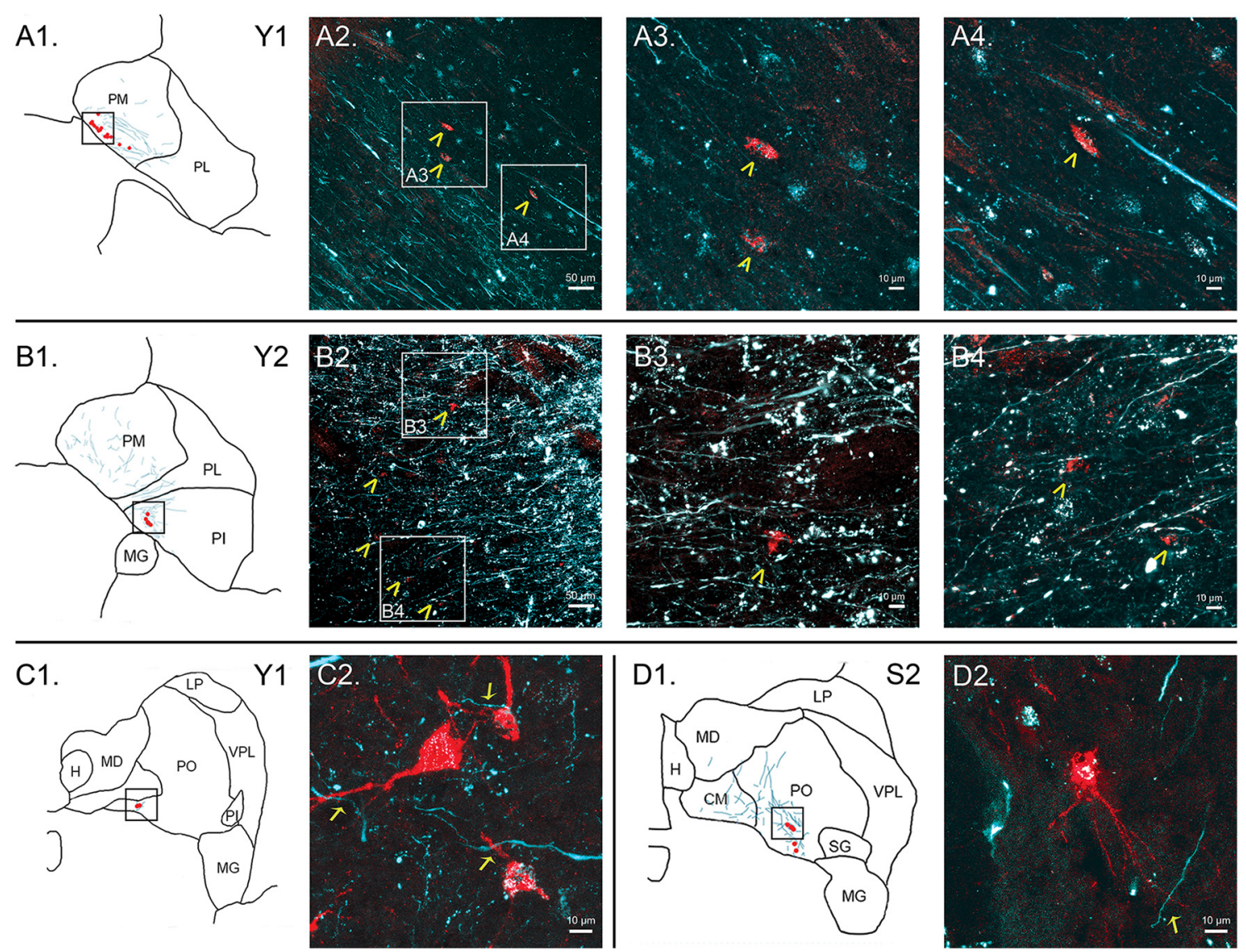

FIGURE 7 | Colocalization of retrograde and anterograde labeling in the pulvinar. FE-labeled fibers resulting from injection into the SC are shown in cyan. CTB-immunoreactive cell bodies resulting from injection into the amygdala are shown in red. Atlas pictures are included to illustrate where each photo was taken. The case from which the images are derived is shown in the upper right corner. (A) Images of cells within PM, case Y1. Atlas image is shown in (A1). Low power confocal image (20x magnification, (A2)) showing a band of labeled cell bodies (red) within the field of labeled axons running along the medial boundary of the PM, parallel to the brachium of the SC. Atlas image is shown in (B1). Chevrons indicate cell bodies. Panels (A3,A4) are higher power $(60 \times)$ magnifications of the areas indicated by boxes in (A2). (B) Images of cells within PI, case Y2. Atlas image is shown in (B1). Low power (20x, (B2)) confocal image showing colocalization of labeled cell bodies and axons in PI. Chevrons indicate cell bodies. Panels (B3, B4) show high power (60x) magnification. As in (A), chevrons in B3 and B4 indicate areas of overlap in labeled cell bodies and axons. (C) Cells within PO, case Y1. Atlas image shown in (C1). High power (63x) confocal image showing individual cell bodies and finer axonal processes in close proximity in (C2). (D) Cells within PO, case S2. Atlas image shown in (D1). High power (63x) confocal image in (D2). Arrows indicate close associations between labeled terminal boutons and labeled cells. Scale bars are as indicated in each panel. 
from the FE injections in SC. Representative labeling is shown in medial (Figure 7A), oral (Figures 7C,D) and inferior (Figure 7B) pulvinar. Low-power $(20 \times)$ magnification shows several choleratoxin-positive cell bodies in close proximity to FE-labeled axons, while high-power (60-63×) magnification shows the processes of cell bodies and axons in close proximity to one another. As described above, labeling of cells projecting from the pulvinar to the amygdala was sparse and ventromedially concentrated, while labeling of axons from SC was ubiquitous in all images. FE-labeled axons in PM followed along the brachium, while labeling within oral and PI was more diffuse. CTB-labeled cells frequently appeared within close proximity of one another.

\section{DISCUSSION}

Here, we have shown a colocalization of projections from the SC with amygdala-projecting neurons in all nuclei of the macaque pulvinar, with the heaviest label occurring ventromedially in oral and PM. The overlapping distributions of retrograde and anterograde label were present in all four cases analyzed. These data provide an anatomical basis for the previously hypothesized subcortical connection between the SC and the amygdala, via the pulvinar. Since we found the same pattern of labeling in the pulvinar in two species of macaques, it is thus likely that these anatomical projections are preserved throughout this genus. While prior studies have suggested the presence of this pathway in primates through MRI-based approaches, these data provide the first direct anatomical evidence for colocalization of these projections within the primate pulvinar.

The cases we presented were tightly clustered and highly overlapping with respect to their injection sites in the amygdala and SC. Despite this there were some observable differences. For example, following the injection of the tracers in the amygdala, labeling in the PO was present in the two cases with more medial placement (Case Y2 and S2) within the lateral nucleus, and absent in the two cases with more lateral placement (Case Y1 and S1). We did not note other patterns with respect to the location of injections within the amygdala. This projection was examined in more detail by others (e.g., Stefanacci and Amaral, 2000). With respect to the injections in the SC, we noted that the two cases with more superficial injections ( $\mathrm{Y} 2$ and S2) were associated with stronger labeling in the PO; we did not note any other patterns. As with the amygdala, the topography of projections from SC to pulvinar were examined in detail by others (Benevento and Standage, 1983; Huerta and Harting, 1983). More detailed assessment of this pathway, with injection sites aimed at the different layers of the SC and different subregions of the amygdala, should be addressed in future studies.

\section{Superior Colliculus Projections to the Pulvinar}

In the rat and mouse, SC projects to several thalamic nuclei, including the LP nucleus (the rodent pulvinar homolog, see
Harting et al., 1972) and the SG nucleus (Taylor et al., 1986; Linke et al., 1999; Zhou et al., 2017). Similarly, the SC projects to the pulvinar of the treeshrew, a proto-primate (Chomsung et al., 2008). This projection is also conserved in primates (May, 2006). In macaques, retrograde tracer injections into PM result in dense labeling of cells within the deep and intermediate layers of SC (Benevento and Standage, 1983). Moreover, lesions made in the superficial, intermediate, and deep layers of the SC result in degenerating fibers in medial and PL (Benevento and Fallon, 1975). Fibers projecting from the SC to pulvinar course laterally from the brachium and terminate throughout all of the subdivisions of the pulvinar of the squirrel monkey, owl monkey and macaque (Benevento and Fallon, 1975; Huerta and Harting, 1983; Stepniewska et al., 2000), a pattern similar to that reported here. Thus, our present findings are in line with those previously reported in the literature.

\section{Pulvinar Projections to the Amygdala}

As with projections from the SC to the pulvinar, projections from the pulvinar to the amygdala have also been described across species. In rodents, for example, the LP nucleus projects to the lateral amygdala (Doron and LeDoux, 1999; Zhou et al., 2018). In macaques, large retrograde (horseradish peroxidase) tracer injections into the amygdala (Aggleton et al., 1980), as well as tracer injections restricted to the lateral nucleus (Norita and Kawamura, 1980; Stefanacci and Amaral, 2000), produce light labeling in the PM, primarily in a region adjacent to the brachium. In the latter study, three cases were presented in which retrograde tracers were placed in either the dorsal division or ventral intermediate division of the lateral nucleus of the amygdala. These cases displayed labeling across the rostrocaudal extend of the medial boundary of the pulvinar, a profile similar to that we observed in our cases S2 and Y2 (Figures 2, 4, 6). These projections have also been confirmed after injections of anterograde tracer into the lateral and PM in both squirrel and macaque monkeys resulted in dense terminal labeling in the dorsal division of the lateral nucleus of the amygdala (Jones and Burton, 1976; Romanski et al., 1997). More restricted injections into the medial division of the PM (i.e., along the brachium of the SC) preferentially label a narrow band at the lateral edge of the lateral nucleus of the amygdala (Burton and Jones, 1976). This pattern of labeling is consistent with what we observed in Case Y1 (Figures 2, 3).

\section{Convergent Labeling Within Pulvinar}

In the mouse, a monosynaptic relay between the lateral SC and the lateral amygdala has been described (Wei et al., 2015). This projection is mediated by a synapse in the LP nucleus of the thalamus, the mouse homolog of the primate pulvinar (Harting et al., 1972). Interestingly, this projection is necessary for species typical responses to looming threatening stimuli (Wei et al., 2015). A similar relationship has been suggested in the rat and the treeshrew (Linke et al., 1999; Day-Brown et al., 2010). Evidence from imaging studies is consistent with these findings. In both macaque and human subjects, reports using DTI suggest that axons run from the SC to the pulvinar, and from the pulvinar 
to the amygdala (Rafal et al., 2015). Tamietto et al. (2012) used DTI to compare human control subjects to a patient with lesions to striate cortex. They found that probabilistic fiber bundles connecting the SC, pulvinar and amygdala exist in both lesioned and control patients, but that the projection is more robust ipsilateral to the damage in striate cortex in the lesioned subject. DTI is a probabilistic technique and cannot conclusively show either functional or anatomical coupling among the areas. Our data fill the gap between the human and primate imaging studies and the rodent data, by confirming that there is an area of overlapping projection in the pulvinar that likely connects the SC with the amygdala.

\section{Relevance to Behavior}

Studies of blindsight have long suggested that SC is necessary for rapid visual processing, by providing an additional subcortical route for information transfer (Schneider, 1969; Weiskrantz et al., 1974). Accordingly, while lesions to striate cortex partially spare performance on visually-mediated tasks (Schmid et al., 2010), lesions or inactivation of both striate cortex and SC produce more profound deficits (Mohler and Wurtz, 1977; Solomon et al., 1981; Kato et al., 2011).

This pathway likely also serves to support rapid, non-conscious perception of threat via projections from the SC to the pulvinar, and from the pulvinar in turn, to the amygdala. Consistent with this, an increase in correlated activation among the amygdala, pulvinar, and SC is observed via fMRI when angry faces are presented in a manner that precludes conscious perception of the stimuli (Morris et al., 1999). The importance of pulvinar in this pathway is further underscored by a finding in a patient with pulvinar damage, who displays impaired fear responses to subliminally presented threatening visual stimuli (Ward et al., 2005).

Studies have also suggested that processing within the pulvinar per se may contribute to the rapid detection of and response to visual threats. Both humans (as measured via MRI) and macaques (as measured by single unit activity) display increased activity in the pulvinar when presented with images of snakes (Van Le et al., 2013; Le et al., 2014, 2016; Almeida et al., 2015; Soares et al., 2017). Of particular relevance to our present findings, there are two apparent clusters of snake-responsive neurons in the macaque pulvinar: one cluster in the dorsal portion of the PL, and the other in the ventromedial portion of the PM. This latter area is where we observed consistent colocalization across all four cases. In their article, Van Le et al. (2013) suggested that at least one route that could mediate rapid responses to threating stimuli such as snakes was a pathway from

\section{REFERENCES}

Aggleton, J. P., Burton, M. J., and Passingham, R. E. (1980). Cortical and subcortical afferents to the amygdala of the rhesus monkey (Macaca mulatta). Brain Res. 190, 347-368. doi: 10.1016/0006-8993(80)90279-6

Almeida, I., Soares, S. C., and Castelo-Branco, M. (2015). The distinct role of the amygdala, superior colliculus and pulvinar in processing of central and peripheral snakes. PLoS One 10:e0141175. doi: 10.1371/journal.pone. 0141175 the SC to the pulvinar to the amygdala; our data are consistent with their interpretation.

Although it is possible that the pulvinar is relaying purely visual information to the amygdala, the intermediate and deep layers of the SC, which we have targeted in this study, receive multimodal input from different sensory systems (Stein et al., 2009). Given that other studies (Benevento and Standage, 1983) have reported preferential labeling in the intermediate and deep SC following injections into the PM, it seems likely that the labeling we observed was also due to projections from the deep and intermediate layers. This would be consistent with a multimodal relay, rather than a purely visual relay. The multimodal integration properties of the intermediate and deep layers of SC may enable its critical role for fast, reflexive defense responses (Dean et al., 1989; Brandão et al., 1994). We have recently reported that these defense responses, which are evoked by pharmacological activation of the primate SC (DesJardin et al., 2013), can be partially attenuated by concurrent inactivation of the basolateral amygdala (Forcelli et al., 2016). However, as no direct projection from SC to amygdala has been reported, it is tempting to speculate that the pulvinar is a component of the network underlying these defense responses.

In summary, here we have described a zone within the pulvinar that receives projections from the SC and contains neurons that project to the amygdala. While such a projection has been hypothesized in humans and macaques, these data provide the first direct anatomical evidence for its existence.

\section{AUTHOR CONTRIBUTIONS}

CE, PF, RS and LM performed experiments, edited the figures and wrote and edited the manuscript. CE analyzed the data and created the figures.

\section{FUNDING}

This work was supported by National Institute of Mental Health R01 MH099505 (LM), National Center for Advancing Translational Sciences KL2 TR001432 (PF) National Institute of Neurological Disorders and Stroke. PF was supported by National Institute of Neurological Disorders and Stroke R01 NS097762. CE was supported by T32 NS041231.

\section{ACKNOWLEDGEMENTS}

We thank Brittany Aguilar, Elyssa LaFlamme, Evan Wicker and Hannah Waguespack for technical support.

Amaral, D. G., Price, J. L., Pitkanen, A., and Carmichael, T. (1992). “Anatomical organization of the primate amygdaloid complex," in The Amygdala: Neurobiological Aspects of Emotion, Memory and Mental Dysfunction, ed. J. Aggleton (New York, NY: Wiley-Liss), 1-66.

Benevento, L. A., and Fallon, J. H. (1975). The ascending projections of the superior colliculus in the rhesus monkey (Macaca mulatta). J. Comp. Neurol. 160, 339-361. doi: 10.1002/cne.901600306

Benevento, L. A., and Standage, G. P. (1983). The organization of projections of the retinorecipient and nonretinorecipient nuclei of the pretectal complex and 
layers of the superior colliculus to the lateral pulvinar and medial pulvinar in the macaque monkey. J. Comp. Neurol. 217, 307-336. doi: 10.1002/cne. 902170307

Born, R. T., and Bradley, D. C. (2005). Structure and function of visual area MT. Annu. Rev. Neurosci. 28, 157-189. doi: 10.1146/annurev.neuro.26.041002. 131052

Brandão, M. L., Cardoso, S. H., Melo, L. L., Motta, V., and Coimbra, N. C. (1994). Neural substrate of defensive behavior in the midbrain tectum. Neurosci. Biobehav. Rev. 18, 339-346. doi: 10.1016/0149-7634(94) 90047-7

Burton, H., and Jones, E. G. (1976). The posterior thalamic region and its cortical projection in new world and old world monkeys. J. Comp. Neurol. 168, 249-301. doi: 10.1002/cne.901680204

Chomsung, R. D., Petry, H. M., and Bickford, M. E. (2008). Ultrastructural examination of diffuse and specific tectopulvinar projections in the tree shrew. J. Comp. Neurol. 510, 24-46. doi: 10.1002/cne.21763

Cowey, A., and Stoerig, P. (1991). The neurobiology of blindsight. Trends Neurosci. 14, 140-145. doi: 10.1016/0166-2236(91)90085-9

Day-Brown, J. D., Wei, H., Chomsung, R. D., Petry, H. M., and Bickford, M. E. (2010). Pulvinar projections to the striatum and amygdala in the tree shrew. Front. Neuroanat. 4:143. doi: 10.3389/fnana.2010.00143

de Gelder, B., Vroomen, J., Pourtois, G., and Weiskrantz, L. (1999). Non-conscious recognition of affect in the absence of striate cortex. Neuroreport 10, 3759-3763. doi: 10.1097/00001756-199912160-00007

Dean, P., Redgrave, P., and Westby, G. W. M. (1989). Event or emergency? Two response systems in the mammalian superior colliculus. Trends Neurosci. 12, 137-147. doi: 10.1016/0166-2236(89)90052-0

DesJardin, J. T., Holmes, A. L., Forcelli, P. A., Cole, C. E., Gale, J. T., Wellman, L. L., et al. (2013). Defense-like behaviors evoked by pharmacological disinhibition of the superior colliculus in the primate. J. Neurosci. 33, 150-155. doi: 10.1523/jneurosci.2924-12.2013

Doron, N. N., and LeDoux, J. E. (1999). Organization of projections to the lateral amygdala from auditory and visual areas of thalamus in the rat. J. Comp. Neurol. 409, 383-409. doi: 10.1002/(sici)1096-9861(19990927)412:3<383::aid-cne2>3. $0 . \operatorname{co} ; 2-5$

Forcelli, P. A., DesJardin, J. T., West, E. A., Holmes, A. L., Elorette, C., Wellman, L. L., et al. (2016). Amygdala selectively modulates defensive responses evoked from the superior colliculus in non-human primates. Soc. Cogn. Affect. Neurosci. 11, 2009-2019. doi: 10.1093/scan/ nsw111

Gattass, R., Soares, J. G., and Lima, B. (2018). "Connectivity of the pulvinar," in The Pulvinar Thalamic Nucleus of Non-Human Primates: Architectonic and Functional Subdivisions, ed. H. W. Korf (Frankfurt: Springer), 19-29.

Gutierrez, C., Yaun, A., and Cusick, C. G. (1995). Neurochemical subdivisions of the inferior pulvinar in macaque monkeys. J. Comp. Neurol. 363, 545-562. doi: 10.1002/cne.903630404

Harting, J. K., Hall, W. C., and Diamond, I. T. (1972). Evolution of the pulvinar. Brain Behav. Evol. 6, 424-452. doi: 10.1159/000123767

Hofbauer, A., and Dräger, U. C. (1985). Depth segregation of retinal ganglion cells projecting to mouse superior colliculus. J. Comp. Neurol. 234, 465-474. doi: 10.1002/cne.902340405

Huerta, M. F., and Harting, J. K. (1983). Sublamination within the superficial gray layer of the squirrel monkey: an analysis of the tectopulvinar projection using anterograde and retrograde transport methods. Brain Res. 261, 119-126. doi: 10.1016/0006-8993(83)91290-8

Humphrey, N. K., and Weiskrantz, L. (1967). Vision in monkeys after removal of the striate cortex. Nature 215, 595-597. doi: 10.1038/215595a0

Jones, E. G., and Burton, H. (1976). A projection from the medial pulvinar to the amygdala in primates. Brain Res. 104, 142-147. doi: 10.1016/00068993(76)90654-5

Kato, R., Takaura, K., Ikeda, T., Yoshida, M., and Isa, T. (2011). Contribution of the retino-tectal pathway to visually guided saccades after lesion of the primary visual cortex in monkeys. Eur. J.Neurosci. 33, 1952-1960. doi: 10.1111/j.14609568.2011.07729.x

Koller, K., Rafal, R. D., Platt, A., and Mitchell, N. D. (2018). Orienting toward threat: contributions of a subcortical pathway transmitting retinal afferents to the amygdala via the superior colliculus and pulvinar. Neuropsychologia doi: 10.1016/j.neuropsychologia.2018.01.027 [Epub ahead of print].
Le, Q. V., Isbell, L. A., Matsumoto, J., Le, V. Q., Nishimaru, H., Hori, E., et al. (2016). Snakes elicit earlier and monkey faces, later, gamma oscillations in macaque pulvinar neurons. Sci. Rep. 6:20595. doi: 10.1038/srep20595

Le, Q. V., Isbell, L. A., Matsumoto, J., Le, V. Q., Hori, E., Maior, R. S., et al. (2014). Monkey pulvinar neurons fire differentially to snake postures. PLoS One 9:e114258. doi: 10.1371/journal.pone.0114258

LeDoux, J. E. (1996). The Emotional Brain: the Mysterious Underpinnings of Emotional Life. (New York, NY: Simon and Schuster).

Liddell, B. J., Brown, K. J., Kemp, A. H., Barton, M. J., Das, P., Peduto, A., et al. (2005). A direct brainstem-amygdala-cortical "alarm" system for subliminal signals of fear. Neuroimage 24, 235-243. doi: 10.1016/j.neuroimage.2004. 08.016

Linke, R., De Lima, A. D., Schwegler, H., and Pape, H.-C. (1999). Direct synaptic connections of axons from superior colliculus with identified thalamo-amygdaloid projection neurons in the rat: possible substrates of a subcortical visual pathway to the amygdala. J. Comp. Neurol. 403, 158-170. doi: 10.1002/(sici)1096-9861(19990111)403:2<158::aid-cne2>3.0.co;2-6

Locke, S. (1960). The projection of the medial pulvinar of the macaque. J. Comp. Neurol. 115, 155-169. doi: 10.1002/cne.901150205

May, P. J. (2006). The mammalian superior colliculus: laminar structure and connections. Prog. Brain Res. 151, 321-378. doi: 10.1016/s0079-6123(05) 51011-2

Miller, M., Pasik, P., and Pasik, T. (1980). Extrageniculostriate vision in the monkey: VII. Contrast sensitivity functions. J. Neurophysiol. 43, 1510-1526. doi: 10.1152/jn.1980.43.6.1510

Mohler, C. W., and Wurtz, R. H. (1977). Role of striate cortex and superior colliculus in visual guidance of saccadic eye movements in monkeys. J. Neurophysiol. 40, 74-94. doi: 10.1152/jn.1977.40.1.74

Morris, J. S., De Gelder, B., Weiskrantz, L., and Dolan, R. J. (2001). Differential extrageniculostriate and amygdala responses to presentation of emotional faces in a cortically blind field. Brain 124, 1241-1252. doi: 10.1093/brain/124.6.1241

Morris, J. S., Ohman, A., and Dolan, R. J. (1999). A subcortical pathway to the right amygdala mediating "unseen" fear. Proc. Natl. Acad. Sci. U S A 96, 1680-1685. doi: 10.1073/pnas.96.4.1680

Norita, M., and Kawamura, K. (1980). Subcortical afferents to the monkey amygdala: an HRP study. Brain Res. 190, 225-230. doi: 10.1016/00068993(80)91171-3

Olszewski, J. (1952). The Thalamus of the Macaca Mulatta: An Atlas for Use With the Stereotaxic Instrument. Basel and New York: Karger.

Perry, V. H., and Cowey, A. (1984). Retinal ganglion cells that project to the superior colliculus and pretectum in the macaque monkey. Neuroscience 12 , 1125-1137. doi: 10.1016/0306-4522(84)90007-1

Pöppel, E., Held, R., and Frost, D. (1973). Residual visual function after brain wounds involving the central visual pathways in man. Nature 243, 295-296. doi: $10.1038 / 243295 \mathrm{a} 0$

Rafal, R. D., Koller, K., Bultitude, J. H., Mullins, P., Ward, R., Mitchell, A. S., et al. (2015). Connectivity between the superior colliculus and the amygdala in humans and macaque monkeys: virtual dissection with probabilistic DTI tractography. J. Neurophysiol. 114, 1947-1962. doi: 10.1152/jn.01016.2014

Richards, W. (1973). Visual processing in scotomata. Exp. Brain Res. 17, 333-347. doi: $10.1007 /$ bf00234098

Romanski, L. M., Giguere, M., Bates, J. F., and Goldman-Rakic, P. S. (1997). Topographic organization of medial pulvinar connections with the prefrontal cortex in the rhesus monkey. J. Comp. Neurol. 379, 313-332. doi: 10.1002/(sici)1096-9861(19970317)379:3<313::aid-cnel>3.0.co;2-6

Rosene, D. L., Roy, N. J., and Davis, B. J. (1986). A cryoprotection method that facilitates cutting frozen sections of whole monkey brains for histological and histochemical processing without freezing artifact. J. Histochem. Cytochem. 34, 1301-1315. doi: 10.1177/34.10.3745909

Sahraie, A., Weiskrantz, L., and Barbur, J. L. (1998). Awareness and confidence ratings in motion perception without geniculo-striate projection. Behav. Brain Res. 96, 71-77. doi: 10.1016/s0166-4328(97)00194-0

Sanders, M. D., Warrington, E., Marshall, J., and Wieskrantz, L. (1974). "Blindsight" vision in a field defect. Lancet 303, 707-708. doi: 10.1016/s01406736(74)92907-9

Saunders, R. C., Aigner, T. G., and Frank, J. A. (1990). Magnetic resonance imaging of the rhesus monkey brain: use for stereotactic neurosurgery. Exp. Brain Res. 81, 443-446. doi: 10.1007/bf00228139 
Schmid, M. C., Mrowka, S. W., Turchi, J., Saunders, R. C., Peters, A. J., Ye, F. Q., et al. (2010). Blindsight depends on the lateral geniculate nucleus. Nature 466, 373-377. doi: 10.1038/nature09179

Schmid, M. C., Panagiotaropoulos, T., Augath, M. A., Logothetis, N. K., and Smirnakis, S. M. (2009). Visually driven activation in macaque areas V2 and V3 without input from the primary visual cortex. PLoS One 4:e5527. doi: 10.1371/journal.pone.0005527

Schneider, G. E. (1969). Two visual systems. Science 163, 895-902. doi: $10.1126 /$ science.163.3870.895

Silverstein, D. N., and Ingvar, M. (2015). A multi-pathway hypothesis for human visual fear signaling. Front. Syst. Neurosci. 9:101. doi: 10.3389/fnsys.2015.00101

Soares, S. C., Maior, R. S., Isbell, L. A., Tomaz, C., and Nishijo, H. (2017). Fast detector/first responder: interactions between the superior colliculuspulvinar pathway and stimuli relevant to primates. Front. Neurosci. 11:67. doi: 10.3389/fnins.2017.00067

Solomon, S. J., Pasik, T., and Pasik, P. (1981). Extrageniculostriate vision in the monkey. VIII. Critical structures for spatial localization. Exp. Brain Res. 44, 259-270. doi: 10.1007/bf00236563

Stefanacci, L., and Amaral, D. G. (2000). Topographic organization of cortical inputs to the lateral nucleus of the macaque monkey amygdala: a retrograde tracing study. J. Comp. Neurol. 421, 52-79. doi: 10.1002/(sici)10969861(20000522)421:1<52::aid-cne4>3.0.co;2-o

Stein, B. E., Stanford, T. R., and Rowland, B. A. (2009). The neural basis of multisensory integration in the midbrain: its organization and maturation. Hear. Res. 258, 4-15. doi: 10.1016/j.heares.2009.03.012

Stepniewska, I., Qi, H.-X., and Kaas, J. H. (2000). Projections of the superior colliculus to subdivisions of the inferior pulvinar in new world and old world monkeys. Vis. Neurosci. 17, 529-549. doi: 10.1017/s0952523800174048

Takakuwa, N., Kato, R., Redgrave, P., and Isa, T. (2017). Emergence of visuallyevoked reward expectation signals in dopamine neurons via the superior colliculus in V1 lesioned monkeys. Elife 6:e24459. doi: 10.7554/elife.24459

Tamietto, M., Pullens, P., De Gelder, B., Weiskrantz, L., and Goebel, R. (2012). Subcortical connections to human amygdala and changes following destruction of the visual cortex. Curr. Biol. 22, 1449-1455. doi: 10.1016/j.cub.2012. 06.006

Taylor, A. M., Jeffery, G., and Lieberman, A. R. (1986). Subcortical afferent and efferent connections of the superior colliculus in the rat and comparisons between albino and pigmented strains. Exp. Brain Res. 62, 131-142. doi: $10.1007 /$ bf00237409

Trojanowski, J. Q., and Jacobson, S. (1975). Peroxidase labeled subcortical afferents to pulvinar in rhesus monkey. Brain Res. 97, 144-150. doi: 10.1016/0006-8993(75)90922-1

Van Le, Q., Isbell, L. A., Matsumoto, J., Nguyen, M., Hori, E., Maior, R. S., et al. (2013). Pulvinar neurons reveal neurobiological evidence of past selection for rapid detection of snakes. Proc. Natl. Acad. Sci. U S A 110, 19000-19005. doi: 10.1073 /pnas. 1312648110
Vuilleumier, P., Armony, J. L., Clarke, K., Husain, M., Driver, J., and Dolan, R. J. (2002). Neural response to emotional faces with and without awareness: event-related fMRI in a parietal patient with visual extinction and spatial neglect. Neuropsychologia 40, 2156-2166. doi: 10.1016/s0028-3932(02) 00045-3

Ward, R., Danziger, S., and Bamford, S. (2005). Response to visual threat following damage to the pulvinar. Curr. Biol. 15, 571-573. doi: 10.1016/j.cub.2005. 01.056

Warner, C. E., Goldschmidt, Y., and Bourne, J. A. (2010). Retinal afferents synapse with relay cells targeting the middle temporal area in the pulvinar and lateral geniculate nuclei. Front. Neuroanat. 4:8. doi: 10.3389/neuro.05.008.2010

Wei, P., Liu, N., Zhang, Z., Liu, X., Tang, Y., He, X., et al. (2015). Processing of visually evoked innate fear by a non-canonical thalamic pathway. Nat. Commun. 6:6756. doi: 10.1038/ncomms7756

Weiskrantz, L. (1963). Contour discrimination in a young monkey with striate cortex ablation. Neuropsychologia 1, 145-164. doi: 10.1016/00283932(63)90004-6

Weiskrantz, L., Warrington, E. K., Sanders, M. D., and Marshall, J. (1974). Visual capacity in the hemianopic field following a restricted occipital ablation. Brain 97, 709-728. doi: 10.1093/brain/97.4.709

Wellman, L. L., Gale, K., and Malkova, L. (2005). GABAA-mediated inhibition of basolateral amygdala blocks reward devaluation in macaques. J. Neurosci. 25, 4577-4586. doi: 10.1523/jneurosci.2257-04.2005

Whalen, P. J., Rauch, S. L., Etcoff, N. L., Mcinerney, S. C., Lee, M. B., and Jenike, M. A. (1998). Masked presentations of emotional facial expressions modulate amygdala activity without explicit knowledge. J. Neurosci. 18, 411-418. doi: 10.1523/jneurosci.18-01-00411.1998

Zhou, N. A., Maire, P. S., Masterson, S. P., and Bickford, M. E. (2017). The mouse pulvinar nucleus: organization of the tectorecipient zones. Vis. Neurosci. 34:E011. doi: 10.1017/s0952523817000050

Zhou, N., Masterson, S. P., Damron, J. K., Guido, W., and Bickford, M. E. (2018). The mouse pulvinar nucleus links the lateral extrastriate cortex, striatum and amygdala. J. Neurosci. 38, 347-362. doi: 10.1523/jneurosci.1279 $-17.2017$

Conflict of Interest Statement: The authors declare that the research was conducted in the absence of any commercial or financial relationships that could be construed as a potential conflict of interest.

Copyright (ㄷ) 2018 Elorette, Forcelli, Saunders and Malkova. This is an open-access article distributed under the terms of the Creative Commons Attribution License (CC BY). The use, distribution or reproduction in other forums is permitted, provided the original author(s) and the copyright owner(s) are credited and that the original publication in this journal is cited, in accordance with accepted academic practice. No use, distribution or reproduction is permitted which does not comply with these terms. 